\title{
Linear-Linear Basis Functions for MLFMA Solutions of Magnetic-Field and Combined-Field Integral Equations
}

\author{
Özgür Ergül, Student Member, IEEE, and Levent Gürel, Senior Member, IEEE
}

\begin{abstract}
We present the linear-linear (LL) basis functions to improve the accuracy of the magnetic-field integral equation (MFIE) and the combined-field integral equation (CFIE) for three-dimensional electromagnetic scattering problems involving closed conductors. We consider the solutions of relatively large scattering problems by employing the multilevel fast multipole algorithm. Accuracy problems of MFIE and CFIE arising from their implementations with the conventional Rao-Wilton-Glisson (RWG) basis functions can be mitigated by using the $L L$ functions for discretization. This is achieved without increasing the computational requirements and with only minor modifications in the existing codes based on the RWG functions.
\end{abstract}

Index Terms-Basis functions, combined-field integral equation, magnetic-field integral equation, multilevel fast multipole algorithm.

\section{INTRODUCTION}

I N THE solution of three-dimensional (3-D) electromagnetic scattering problems by the method of moments (MOM) [1], it is common to apply a triangulation on the scatterer and employ Rao-Wilton-Glisson (RWG) [2] basis functions defined on planar triangles to expand the unknown surface current density. The scattering problem can be formulated either as an electric-field integral equation (EFIE) or as a magnetic-field integral equation (MFIE) [3]. Although the same physical current distribution is expanded by an identical set of RWG functions in both cases, the same levels of accuracy cannot be obtained by EFIE and MFIE. Independent of the shape of the geometry (smooth or sharp), MFIE with the RWG functions has a consistent error that produces worse results for radar cross section (RCS) than EFIE [4].

We recently conducted a series of studies to investigate in detail the MFIE inaccuracy [4]-[7], and systematically compared the EFIE and MFIE implementations in order to identify the source of the error. In those studies the following applies.

1) The singularities arising in the outer integrals of MFIE were extracted for improved accuracy in the numerical integrations [5];

Manuscript received April 21, 2006; revised November 20, 2006. This work was supported in part by the Scientific and Technical Research Council of Turkey (TUBITAK) under Research Grant 105E172, in part by the Turkish Academy of Sciences in the framework of the Young Scientist Award Program (LG/TUBA-GEBIP/2002-1-12), and in part by contracts from ASELSAN and SSM.

The authors are with the Department of Electrical and Electronics Engineering, Bilkent University, TR-06800 Bilkent, Ankara, Turkey (e-mail: ergul@ee.bilkent.edu.tr, 1gurel@bilkent.edu.tr).

Digital Object Identifier 10.1109/TAP.2007.893393
2) The testing scheme of MFIE was extended by allowing the testing points to reside on the edges of the triangulation [6];

3) The solid-angle factor in MFIE was clarified to avoid incorrect usage [7].

Although these studies provided a better understanding of MFIE and led to more robust implementations, the improvement in the accuracy was limited, indicating that the fundamental reason for the inaccuracy lied elsewhere. Then, we focused on the modeling of the current distribution with different basis functions. By testing the accuracy of MFIE with respect to the basis and testing functions [8], we demonstrated that the accuracy of MFIE can be significantly improved by employing more suitable functions rather than the RWG functions [9]. This is also confirmed by others [10]-[13].

In this paper, we present the linear-linear (LL) basis functions to further improve the accuracy of the MFIE and CFIE implementations. These functions were employed previously for EFIE [14]. We also employed them for MFIE [15] and demonstrated that the LL functions improve the accuracy of MFIE significantly for moderate-size problems, including those with sharp edges and corners. In this paper, we consider the solutions of large problems with the multilevel fast multipole algorithm (MLFMA) [16]. We show that the RCS results obtained by the MFIE and CFIE solutions are inaccurate even for large and smooth geometries, such as a sphere, compared to the solutions by EFIE. The accuracy can be improved with the LL functions without increasing the computational requirements.

The LL functions can also be identified as a subset of the LN/QT or QN/LT type vector functions discussed in [17]. Contrary to the RWG functions, LL functions are first-order complete to represent the vectors by providing six degrees of freedom to model the linear variation on the triangles. As depicted in Fig. 1, there are two kinds of LL functions defined on the same edge simultaneously to expand the current density better than the RWG functions that are only zeroth-order complete. Improved current modeling with higher-order completeness is achieved at the cost of doubling the number of basis functions compared to the RWG functions for the same triangulation of the geometry. On the other hand, we show that the LL functions provide more accurate RCS results with MFIE and CFIE for the same number of unknowns as the RWG functions. The improvement is present even for the curved geometries, where the discretization for the LL functions is coarser and leads to more deviation from the actual geometry to keep the number of unknowns approximately the same as when the RWG functions are used on the same geometry with a finer triangulation. 


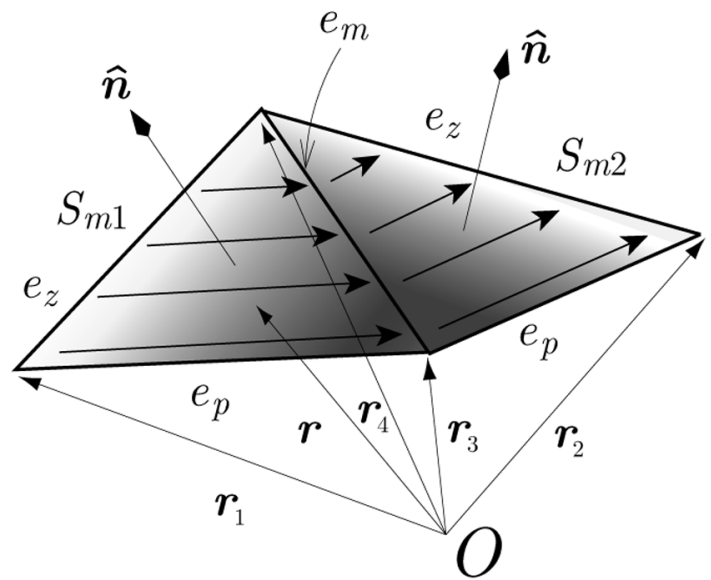

(a)

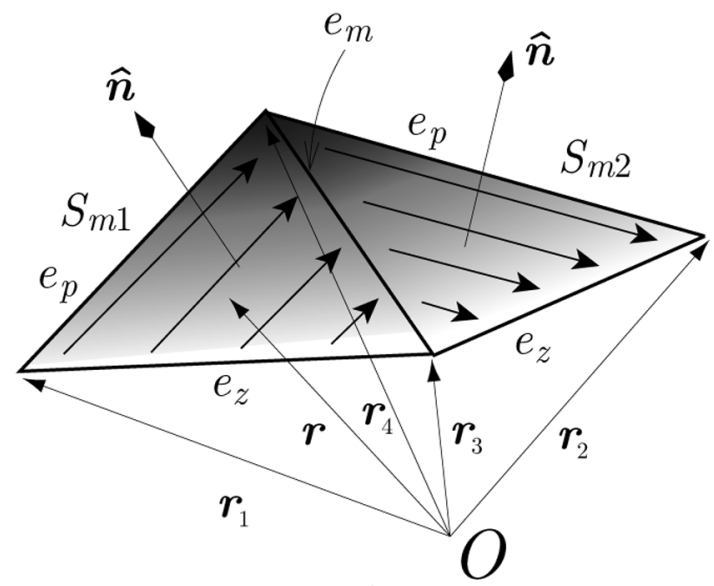

(b)

Fig. 1. (a) First-kind and (b) second-kind LL functions defined on the edge $e_{m}$. The arrows show the direction while the shading indicates the magnitude of the vector distribution. Light and dark colors represent the low and high values, respectively, and white corresponds to zero.

Although the LL functions are first-order complete to represent the vectors, they are only zeroth-order complete to represent the divergence or curl of a vector. In fact, they are simply the decomposition of the RWG functions as elucidated in Section III. The LL functions improve the accuracy of MFIE to the level of EFIE, while they are similar to the RWG functions by providing linear variations for the induced current on the triangular domains. In this manner, the LL functions are different from the higher-order techniques in computational electromagnetics appeared in the literature to improve the accuracy of the integral equations [18]-[20]. The advantages of the LL functions can be listed as follows.

1) The LL functions can be implemented with simple modifications on the existing codes employing the RWG functions. Most of the numerical procedures in MLFMA, such as the singularity extractions for the near-field interactions and Fourier transforms for the far-field interactions, can be adapted from those of the RWG functions;

2) For the same number of unknowns, employing the LL functions in the MLFMA implementations does not require extra computational load compared to employing the RWG functions.
For the solution of large problems, the combined-field integral equation (CFIE) [3] is preferred whenever it is applicable, i.e., when the scatterer is smooth and it can be modelled with a closed conductor. This is because CFIE provides better-conditioned matrix equations compared to EFIE and MFIE [21], and this is essential for iterative solvers such as MLFMA. In addition to this, CFIE is free of the internal-resonance problem by providing unique solutions at the resonance frequencies of EFIE and MFIE [22]. In the case of MFIE with the RWG functions, there are two significant error sources in the computation of the scattered fields, namely, the internal resonance and the inadequate ability of the RWG functions to discretize the MFIE kernel. For CFIE, the first error disappears but the second error due to the RWG functions remains through the contribution of MFIE in CFIE. Therefore, the improved accuracy of MFIE with the new set of basis functions is also essential to obtain accurate results with CFIE.

Finally, for 2-D implementations, it is reported that the error in the MFIE is due to the identity operator and that it can be eliminated by a regularization method [23], [24]. In the present work, we propose a complete change in the set of the basis functions. Extending the regularization to the 3-D MFIE with the RWG functions is more difficult to achieve but it is still under investigation.

\section{DISCRETIZATION}

We consider scattering problems involving 3-D perfectly conducting closed bodies in free space. With the application of the MOM discretization to the integral equations, $N \times N$ matrix equations

$$
\sum_{n=1}^{N} Z_{m n}^{E, M, C} a_{n}=v_{m}^{E, M, C}, \quad m=1, \ldots, N
$$

are obtained. In the above, $a_{n}$ represents the unknown coefficients of the basis functions and $Z_{m n}^{E, M, C}$ represents the matrix elements derived as

$$
\begin{aligned}
& Z_{m n}^{E}=\int_{S_{m}} d \boldsymbol{r} \boldsymbol{t}_{m}(\boldsymbol{r}) \cdot \int_{S_{n}} d \boldsymbol{r}^{\prime} g\left(\boldsymbol{r}, \boldsymbol{r}^{\prime}\right) \boldsymbol{b}_{n}\left(\boldsymbol{r}^{\prime}\right) \\
& \quad-\frac{1}{k^{2}} \int_{S_{m}} d \boldsymbol{r}\left[\nabla \cdot \boldsymbol{t}_{m}(\boldsymbol{r})\right] \int_{S_{n}} d \boldsymbol{r}^{\prime} g\left(\boldsymbol{r}, \boldsymbol{r}^{\prime}\right)\left[\nabla^{\prime} \cdot \boldsymbol{b}_{n}\left(\boldsymbol{r}^{\prime}\right)\right]
\end{aligned}
$$

for EFIE and

$$
\begin{aligned}
Z_{m n}^{M}= & \int_{S_{m}} d \boldsymbol{r} \boldsymbol{t}_{m}(\boldsymbol{r}) \cdot \boldsymbol{b}_{n}(\boldsymbol{r}) \\
& -\int_{S_{m}} d \boldsymbol{r} \boldsymbol{t}_{m}(\boldsymbol{r}) \cdot \hat{\boldsymbol{n}} \times \int_{S_{n}} d \boldsymbol{r}^{\prime} \boldsymbol{b}_{n}\left(\boldsymbol{r}^{\prime}\right) \times \nabla^{\prime} g\left(\boldsymbol{r}, \boldsymbol{r}^{\prime}\right)
\end{aligned}
$$

for MFIE, where

$$
g\left(\boldsymbol{r}, \boldsymbol{r}^{\prime}\right)=\frac{e^{i k R}}{4 \pi R} \quad\left(R=\left|\boldsymbol{r}-\boldsymbol{r}^{\prime}\right|\right)
$$

denotes the free-space Green's function in phasor notation with the $e^{-i w t}$ convention and $k$ is the wavenumber. In (2) and (3), 
$\boldsymbol{b}_{n}$ and $\boldsymbol{t}_{m}$ represent the set of basis and testing functions, respectively, where $n, m=1,2, \ldots, N$ and $N$ is the number of unknowns. The right-hand-side vector in (1) is derived as

$$
v_{m}^{E}=\frac{i}{k \eta} \int_{S_{m}} d \boldsymbol{r} \boldsymbol{t}_{m}(\boldsymbol{r}) \cdot \boldsymbol{E}^{\boldsymbol{i}}(\boldsymbol{r})
$$

and

$$
v_{m}^{M}=\int_{S_{m}} d \boldsymbol{r} \boldsymbol{t}_{m}(\boldsymbol{r}) \cdot \hat{\boldsymbol{n}} \times \boldsymbol{H}^{\boldsymbol{i}}(\boldsymbol{r})
$$

for EFIE and MFIE, respectively, where $\boldsymbol{E}^{\boldsymbol{i}}$ and $\boldsymbol{H}^{\boldsymbol{i}}$ are the incident fields and $\eta$ is the characteristic impedance of free space. Finally, CFIE is obtained by the convex combination of EFIE and MFIE as [25]

$$
\begin{aligned}
Z_{m n}^{C} & =\alpha Z_{m n}^{E}+(1-\alpha) \frac{i}{k} Z_{m n}^{M} \\
v_{m}^{C} & =\alpha v_{m}^{E}+(1-\alpha) \frac{i}{k} v_{m}^{M} .
\end{aligned}
$$

\section{LINEAR-LINEAR FUNCTIONS}

Spatial distributions of the LL functions are depicted in Fig. 1, where there are two kinds of LL functions defined on the same edge $e_{m}$. The expressions for the LL functions of the first kind and second kind are given in (8) and (9), respectively, at the bottom of the page. In (8) and (9), $l_{m}$ represents the length of edge $e_{m}, \hat{\boldsymbol{n}}$ is the outwardly directed normal, and $A_{m 1}$ and $A_{m 2}$ are respectively the areas of the first $\left(S_{m 1}\right)$ and the second $\left(S_{m 2}\right)$ triangles associated with the edge $e_{m}$. In Fig. 1, the vertex locations $\boldsymbol{r}_{1}, \boldsymbol{r}_{2}, \boldsymbol{r}_{3}$, and $\boldsymbol{r}_{4}$ are labelled as well as the three edges of the triangles $e_{m}, e_{p}$, and $e_{z}$ depending on the kind of the LL function. For both kinds of the LL functions, the spatial distribution

1) is parallel to one of the side edges $e_{p}$ over both of the triangles;
2) changes linearly along the edge $e_{p}$ and becomes maximum at the intersection of that edge with the main edge $e_{m}$;

3 ) is exactly zero at the other side edge $e_{z}$;

4) has a continuous normal component across the main edge $e_{m}$

5) varies linearly at the main edge $e_{m}$ in both the perpendicular and tangential directions.

Because of the final property above, we call these functions the linear-linear type, providing linear variation along the main edge in both directions. We note that the RWG functions are constant-linear type since the perpendicular component along the main edge is constant [2].

The LL functions can actually decompose the corresponding RWG function as

$$
\boldsymbol{f}_{m}^{R}(\boldsymbol{r})=\boldsymbol{f}_{m}^{L, 1}(\boldsymbol{r})+\boldsymbol{f}_{m}^{L, 2}(\boldsymbol{r})
$$

where $f_{m}^{R}$ represents the RWG function associated with the $m$ th edge. The charge distribution implied by the expressions in (8) and (9) is

$$
\nabla_{S} \cdot \boldsymbol{f}_{m}^{L, 1}(\boldsymbol{r})=\nabla_{S} \cdot \boldsymbol{f}_{m}^{L, 2}(\boldsymbol{r})= \begin{cases}\frac{l_{m}}{2 A_{m 1}}, & r \in S_{m 1} \\ \frac{-l_{m}}{2 A_{m 2}}, & r \in S_{m 2} \\ 0, & \text { otherwise }\end{cases}
$$

which is constant and exactly half of that for the RWG functions [2]. Equation (11) indicates that the LL functions are divergence-conforming since their divergence is finite. Although this property is not crucial for MFIE, which also allows the use of the curl-conforming functions depending on the formulation type [5], divergence-conforming property of the LL functions becomes essential for the CFIE implementations, where EFIE requires the evaluation of (2).

In the MLFMA implementations, far-field interactions are calculated in a group-by-group manner, as presented in the Appendix. However, the near-field interactions are calculated directly by evaluating the double integrals in (2) and (3). Similar expressions have been investigated in various references

$$
\boldsymbol{f}_{m}^{L, 1}(\boldsymbol{r})= \begin{cases}\frac{l_{m}}{4\left(A_{m 1}\right)^{2}}\left(\boldsymbol{r}-\boldsymbol{r}_{1}\right) \cdot\left[\left(\boldsymbol{r}_{4}-\boldsymbol{r}_{1}\right) \times \hat{\boldsymbol{n}}\right]\left(\boldsymbol{r}_{3}-\boldsymbol{r}_{1}\right), & r \in S_{m 1} \\ \frac{l_{m}}{4\left(A_{m 2}\right)^{2}}\left(\boldsymbol{r}-\boldsymbol{r}_{2}\right) \cdot\left[\left(\boldsymbol{r}_{4}-\boldsymbol{r}_{2}\right) \times \hat{\boldsymbol{n}}\right]\left(\boldsymbol{r}_{3}-\boldsymbol{r}_{2}\right), & r \in S_{m 2} \\ 0, & \text { otherwise }\end{cases}
$$

$$
\boldsymbol{f}_{m}^{L, 2}(\boldsymbol{r})= \begin{cases}\frac{l_{m}}{4\left(A_{m 1}\right)^{2}}\left(\boldsymbol{r}-\boldsymbol{r}_{1}\right) \cdot\left[\left(\boldsymbol{r}_{3}-\boldsymbol{r}_{1}\right) \times \hat{\boldsymbol{n}}\right]\left(\boldsymbol{r}_{4}-\boldsymbol{r}_{1}\right), & r \in S_{m 1} \\ \frac{l_{m}}{4\left(A_{m 2}\right)^{2}}\left(\boldsymbol{r}-\boldsymbol{r}_{2}\right) \cdot\left[\left(\boldsymbol{r}_{3}-\boldsymbol{r}_{2}\right) \times \hat{\boldsymbol{n}}\right]\left(\boldsymbol{r}_{4}-\boldsymbol{r}_{2}\right), & r \in S_{m 2} \\ 0, & \text { otherwise }\end{cases}
$$


[26]-[28]. In general, examination of (2) and (3) using the definitions in (8) and (9) shows that two different types of inner integrals, i.e.

$$
\begin{gathered}
I_{1}=\int_{S_{t}} d \boldsymbol{r}^{\prime}\left(\begin{array}{c}
1 \\
x^{\prime} \\
y^{\prime}
\end{array}\right) g\left(\boldsymbol{r}, \boldsymbol{r}^{\prime}\right) \\
\boldsymbol{I}_{2}=\int_{S_{t}} d \boldsymbol{r}^{\prime}\left(\begin{array}{c}
1 \\
x^{\prime} \\
y^{\prime}
\end{array}\right) \nabla^{\prime} g\left(\boldsymbol{r}, \boldsymbol{r}^{\prime}\right)
\end{gathered}
$$

need to be evaluated, where $S_{t}$ represents the surface of the triangle. We assume that a coordinate transformation is applied so that the triangle is on the $x-y$ plane having a normal in the $z$ direction. For the numerical evaluation of the integral in (12), the singularity can be extracted and the integral can be rewritten as

$I_{1}=\int_{S_{t}} d \boldsymbol{r}^{\prime}\left(\begin{array}{c}1 \\ x^{\prime} \\ y^{\prime}\end{array}\right)\left(g\left(\boldsymbol{r}, \boldsymbol{r}^{\prime}\right)-\frac{1}{4 \pi R}\right)+\int_{S_{t}} d \boldsymbol{r}^{\prime}\left(\begin{array}{c}1 \\ x^{\prime} \\ y^{\prime}\end{array}\right) \frac{1}{4 \pi R}$

where the second term of the right-hand-side is evaluated analytically [26]. We note that

$$
\lim _{R \rightarrow 0}\left(g\left(\boldsymbol{r}, \boldsymbol{r}^{\prime}\right)-\frac{1}{4 \pi R}\right)=\frac{i k}{4 \pi}
$$

and the first term of the right-hand side in (14) can be evaluated numerically using a Gaussian quadrature rule [29] or an adaptive integration method [30].

For the integral in (13), the singularity can be extracted similarly as

$$
\begin{aligned}
\boldsymbol{I}_{2}=\int_{S_{t}} d \boldsymbol{r}^{\prime}\left(\begin{array}{c}
1 \\
x^{\prime} \\
y^{\prime}
\end{array}\right) \nabla^{\prime} & \left(g\left(\boldsymbol{r}, \boldsymbol{r}^{\prime}\right)-\frac{1}{4 \pi R}\right) \\
& +\int_{S_{t}} d \boldsymbol{r}^{\prime}\left(\begin{array}{c}
1 \\
x^{\prime} \\
y^{\prime}
\end{array}\right) \nabla^{\prime}\left(\frac{1}{4 \pi R}\right) .
\end{aligned}
$$

It can be shown that

$$
\lim _{R \rightarrow 0} \nabla^{\prime}\left(g\left(\boldsymbol{r}, \boldsymbol{r}^{\prime}\right)-\frac{1}{4 \pi R}\right)=\frac{\hat{R} k^{2}}{8 \pi}
$$

where $\hat{\boldsymbol{R}}$ is the unit vector defined as

$$
\hat{\boldsymbol{R}}=\frac{\boldsymbol{R}}{R}=\frac{\boldsymbol{r}-\boldsymbol{r}^{\prime}}{\left|\boldsymbol{r}-\boldsymbol{r}^{\prime}\right|}
$$

and the first term of the right-hand-side in (16) is again evaluated numerically, while the second term can be evaluated analytically [27]. The integration in (16) appears in MFIE, which does not require the evaluation of the inner integrals for the self-interactions of the half basis and testing functions on the triangles. Therefore, the discontinuity in the limit shown in (17) does not pose a numerical problem for the calculations of the inner integrals. However, to increase the efficiency and obtain higher accuracy, it is expedient to evaluate the inner integrals in (13) as

$$
\begin{aligned}
\boldsymbol{I}_{2}= & \int_{S_{t}} d \boldsymbol{r}^{\prime}\left(\begin{array}{c}
1 \\
x^{\prime} \\
y^{\prime}
\end{array}\right) \nabla^{\prime}\left(g\left(\boldsymbol{r}, \boldsymbol{r}^{\prime}\right)-\frac{1}{4 \pi R}+\frac{k^{2}}{8 \pi} R\right) \\
& +\int_{S_{t}} d \boldsymbol{r}^{\prime}\left(\begin{array}{c}
1 \\
x^{\prime} \\
y^{\prime}
\end{array}\right) \nabla^{\prime}\left(\frac{1}{4 \pi R}\right)-\frac{k^{2}}{8 \pi} \int_{S_{t}} d \boldsymbol{r}^{\prime}\left(\begin{array}{c}
1 \\
x^{\prime} \\
y^{\prime}
\end{array}\right) \nabla^{\prime} R
\end{aligned}
$$

by extracting two terms from the Green's function [28]. The last term of the right-hand-side in (19) can also be evaluated analytically [26].

\section{RESULTS}

First, we consider scattering problems involving a moderately large sphere of radius $a=6 \lambda$. The sphere is illuminated by a plane wave and the scattering problems discretized by various triangulations are solved by different MLFMA implementations involving the MFIE and CFIE formulations and the RWG and LL basis functions. In Fig. 2(a), normalized forward-scattered RCS is plotted with respect to the number of unknowns. Since the size of the scatterer is fixed with respect to the wavelength, the number of unknowns is controlled by changing the fineness of the triangulation. As the number of unknowns increases from about 50,000 to 500,000, all the computational curves approach the analytical value obtained by a Mie-series solution. Convergence is significantly faster for both the MFIE and CFIE formulations with the LL functions, and MFIE with the RWG functions has the slowest convergence. We also observe the poor performance of CFIE with the RWG functions. Since CFIE is free of any internal-resonance problem, this shows the insufficiency of the RWG functions in the discretization of the MFIE kernel compared to the LL functions.

In Fig. 2(a), the comparison of the RWG and LL functions is performed in a fair manner since the $x$ axis is based on the number of unknowns and not on the mesh size. In fact, the results corresponding to the $\lambda / 10$ mesh size are indicated by dots on the curves; these dots establish that the number of unknowns is doubled in the LL case compared to the RWG case. On the other hand, for the same number of unknowns, the triangulation for the LL functions is coarser than the triangulation for the RWG functions. Coarser mesh leads to more deviation from the actual geometry for curved surfaces, such as the sphere. Consequently, we obtain improvement with the LL functions despite a decrease in the quality of the geometry modeling. Since the computational resources required for the RWG and LL functions are similar for a fixed number of unknowns, the LL functions are superior to RWG functions because of the significant boost in the accuracy.

Fig. 2(b) offers another view for the accuracy of the RCS results obtained with the RWG and LL functions. The RCS error, which is defined as the error in the scattered field in reference to the exact Mie-series solution, is plotted on a $\log -\log$ scale as a function of the number of linear unknowns per wavelength. Then, the slope of the error curve signifies the order of convergence of the numerical solution. It is seen 


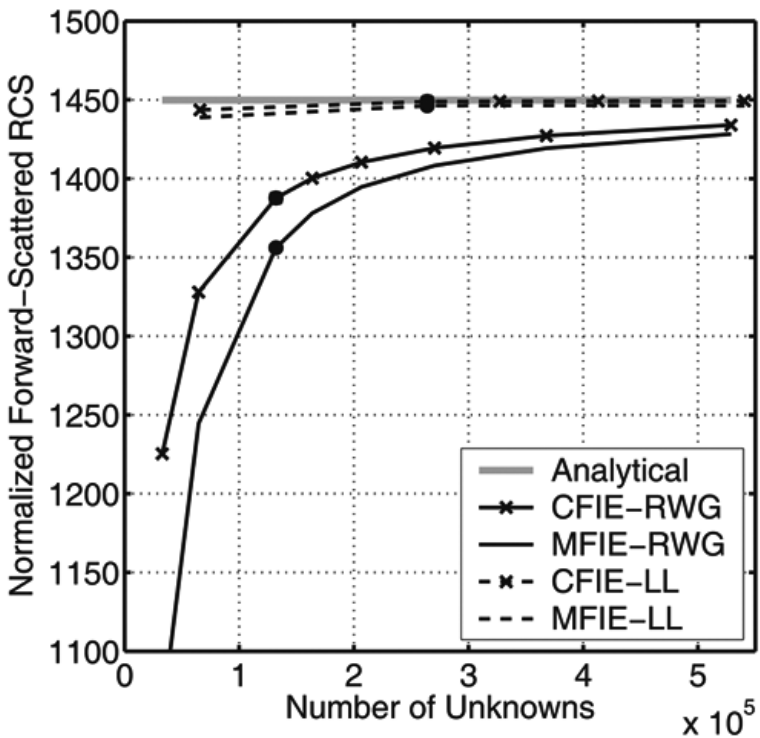

(a)

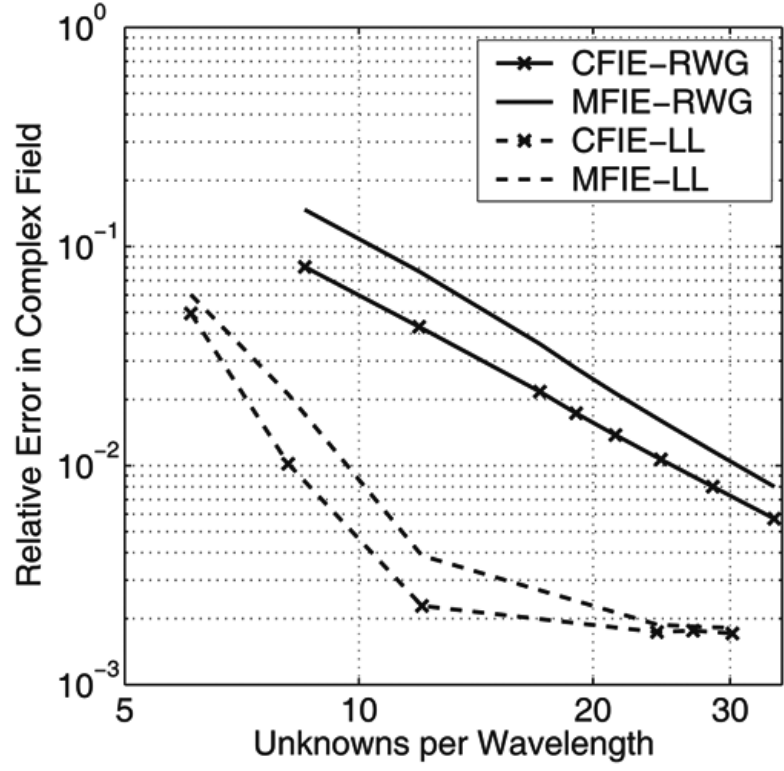

(b)

Fig. 2. (a) Normalized forward-scattered RCS (RCS $\left./ \pi a^{2}\right)$ of a sphere of radius $a=6 \lambda$ with respect to the number of unknowns. Results are obtained by MFIE-RWG, CFIE-RWG, MFIE-LL, CFIE-LL, and a Mie-series solution. Dots on the computational curves represent the triangulations with the mesh size of about $\lambda / 10$. (b) Log-log plot of the error in the scattered field as a function of the number of linear unknowns per wavelength. Slopes of the error curves signify the orders of convergence.
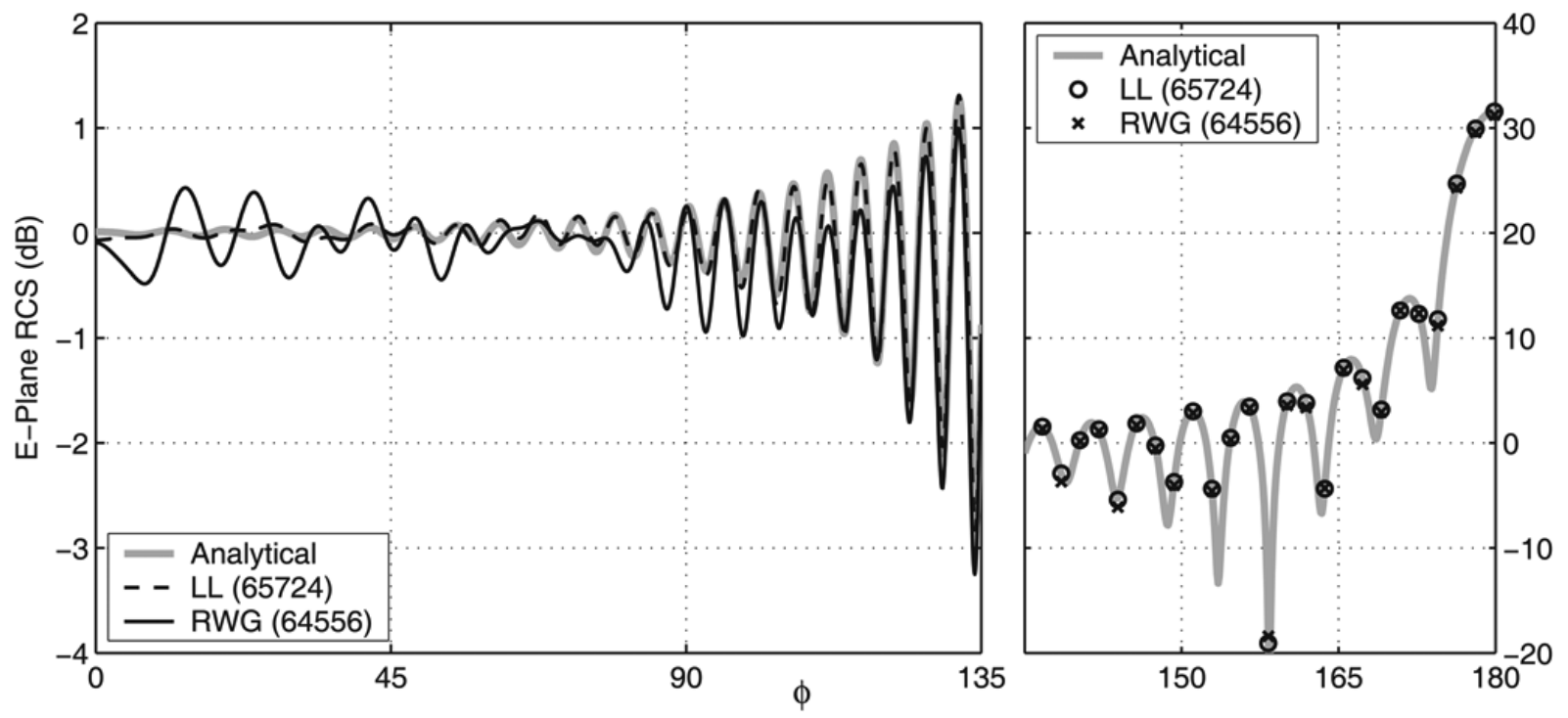

Fig. 3. Bistatic RCS in $\mathrm{dB}$ of a sphere of radius $a=6 \lambda$ on the E-plane with respect to bistatic direction from $0^{\circ}$ (backscattering) to $180^{\circ}$ (forward-scattering). The curves are obtained by the CFIE implementations employing the RWG and LL functions. The Mie-series result is also plotted for comparisons.

in Fig. 2(b) that the error decreases with higher slopes for the LL functions than for the RWG functions, demonstrating that the LL functions provide higher-order convergence. Note that the slopes of the error curves for the LL functions are not constant, as opposed to the slopes of the error curves for the RWG functions. This is because the LL functions reduce the error so effectively that the residual error is mainly due to the discretization, i.e., the error in the geometrical representation of the sphere.

In Fig. 3, we present the bistatic RCS in decibels $(\mathrm{dB})$ on the E-plane. The sphere is discretized with mesh sizes of about $\lambda / 8$ for RWG and $\lambda / 5$ for LL. Then, the numbers of unknowns are approximately the same for the RWG and LL cases although the triangulation is coarser for the LL case as discussed above. In Fig. 3, computational results are obtained with the CFIE formulation, and the analytical results are also displayed. The plot is divided into two parts with different scales of the $y$ axis for clarity of the details. For the bistatic directions from $0^{\circ}$ (backscattering) to $135^{\circ}$, the LL functions unambiguously provide more accurate results. In the range from $135^{\circ}$ to $180^{\circ}$ (forward-scattering), the improvement is not evident in the $\mathrm{dB}$ scale. On the other hand, we plot the relative error in Fig. 4, 


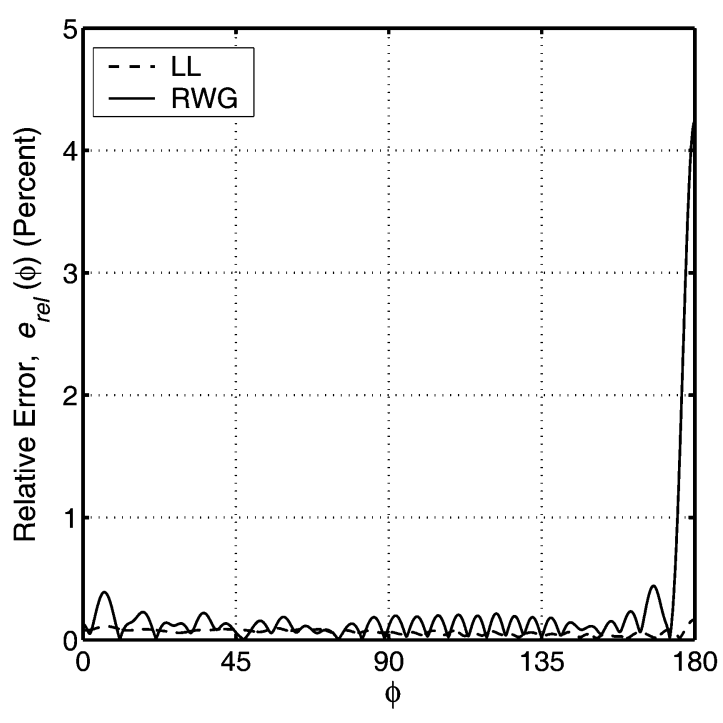

Fig. 4. Relative error as defined in (20) for the bistatic RCS depicted in Fig. 3. The error for the RWG functions is significantly larger than the error for the LL functions, especially in the forward-scattering direction, where the RWG error is above $4 \%$.

where the large error in the forward-scattering direction is visible. We define the relative error as

$$
e_{r e l}(\phi)=\frac{\left|\boldsymbol{E}_{a}(\phi)-\boldsymbol{E}_{c}(\phi)\right|}{\max _{\phi}\left|\boldsymbol{E}_{a}(\phi)\right|}
$$

where $\boldsymbol{E}_{a}$ and $\boldsymbol{E}_{c}$ are the complex-valued scattered fields in the far zone that are obtained analytically (reference) and computationally, respectively. The error with the LL functions is consistently lower and under $0.2 \%$. The error with the RWG functions peaks in the forward-scattering direction, where it is above $4 \%$. This error is not acceptable since the near-field and far-field interactions of the matrix equation are calculated with a maximum error of $1 \%$ and $0.1 \%$, respectively. Furthermore, the relative residual error in the iterative solution is $10^{-6}$ so that we expect the total computational error to be $1 \%$ at most. In the RWG case, the error due to the simultaneous discretization of the geometry and the current distribution is significant and dominates the computational error. As Fig. 4 suggests, the excessive error due to the discretization with the RWG functions can be easily prevented by replacing them with the LL functions.

Finally, we present the results of scattering problems involving a relatively long object with sharp edges and corners as described in Fig. 5. The scatterer is composed of planar surfaces only, and therefore, its triangulation does not lead to a deviation from the actual geometry, as opposed to the sphere. The object is illuminated by a plane wave propagating in the $-y$ direction and we calculate the forward-scattered RCS at $4 \mathrm{GHz}$ by employing the MFIE and CFIE formulations with the RWG and LL functions. We apply different discretizations with the triangulation size varying from 1.5 to $0.375 \mathrm{~cm}$, leading to total of 15,340 to 238,672 triangles, respectively. We also note that $\lambda / 10$ triangulation corresponds to 91,782 edges on the object. Fig. 6 presents the forward-scattered RCS values with

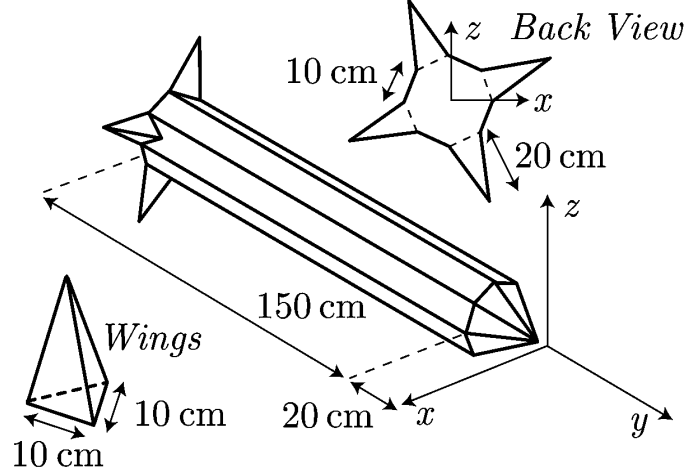

Fig. 5. A scatterer composed of planar surfaces connected with sharp edges and corners.

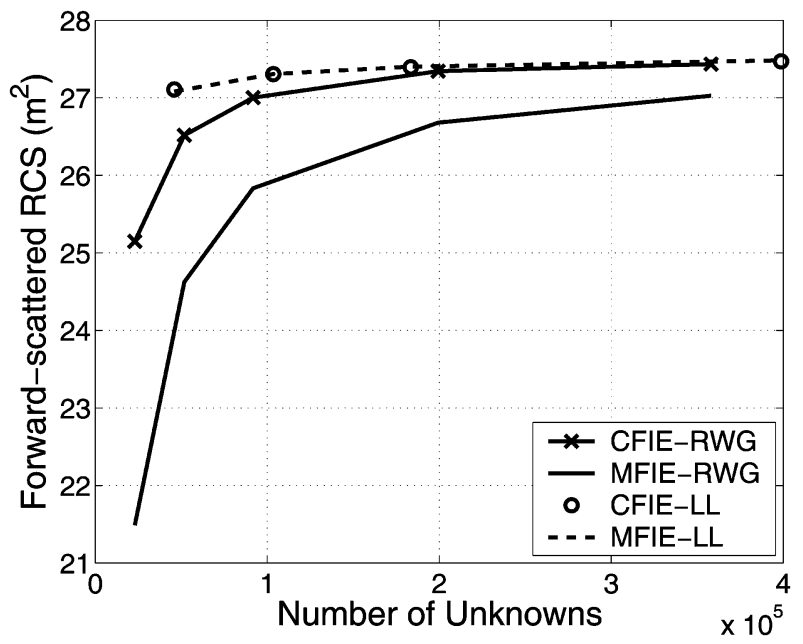

Fig. 6. Forward-scattered RCS values $\left(\mathrm{m}^{2}\right)$ with respect to the number of unknowns for the scatterer in Fig. 5 illuminated by a plane wave propagating in the $-y$ direction at $4 \mathrm{GHz}$. The curves are obtained with the CFIE and MFIE implementations employing the RWG and LL functions.

respect to number of unknowns; we observe that all four curves tend to converge to each other as the mesh gets denser, the number of unknowns increases, and the computations become more accurate. However, the convergence rate depends on the type of the formulation and the basis function. MFIE with the RWG functions has the slowest convergence while CFIE with the RWG functions shows relatively poor performance compared to the MFIE and CFIE formulations employing the LL functions. As in the case of the sphere problem, we observe that the LL functions provide significantly improved accuracy for both MFIE and CFIE solutions of the problem.

\section{CONCLUSION}

The significant error in MFIE and CFIE due to the RWG functions can be easily avoided by employing the LL functions for these equations. Accuracy is improved without increasing the computational costs. Since the LL functions are strongly related to the RWG functions, they can be implemented with simple modifications on the existing codes employing the RWG functions. 


\section{APPENDIX \\ EMPLOYING THE LL FUNCTIONS IN MLFMA}

For the far-field interactions in MLFMA, the matrix elements are derived as [25]

$$
Z_{m n}^{E}=\frac{i k}{(4 \pi)^{2}} \int d^{2} \hat{\boldsymbol{k}} \boldsymbol{F}_{C m}^{E, \operatorname{rec}}(\hat{\boldsymbol{k}}) T_{L}(k,|\boldsymbol{D}|, \hat{\boldsymbol{D}} \cdot \hat{\boldsymbol{k}}) \cdot \boldsymbol{F}_{C^{\prime} n}^{E, \mathrm{rad}}(\hat{\boldsymbol{k}})
$$

for EFIE and

$$
Z_{m n}^{M}=\frac{k^{2}}{(4 \pi)^{2}} \int d^{2} \hat{\boldsymbol{k}} \boldsymbol{F}_{C m}^{M, \text { rec }}(\hat{\boldsymbol{k}}) T_{L}(k,|\boldsymbol{D}|, \hat{\boldsymbol{D}} \cdot \hat{\boldsymbol{k}}) \cdot \boldsymbol{F}_{C^{\prime} n}^{M, \operatorname{rad}}(\hat{\boldsymbol{k}})
$$

for MFIE, where $\hat{\boldsymbol{k}}$ is the angular direction on the unit sphere, and

$$
T_{L}(k,|\boldsymbol{D}|, \hat{\boldsymbol{D}} \cdot \hat{\boldsymbol{k}})=\sum_{l=0}^{L} i^{l}(2 l+1) h_{l}^{(1)}(k D) P_{l}(\hat{\boldsymbol{D}} \cdot \hat{\boldsymbol{k}})
$$

is the translation function written in terms of the spherical Hankel function of the first kind $h_{l}^{(1)}$ and Legendre polynomial $P_{l}$. In (21) and (22), $\boldsymbol{F}_{C m}^{E, \text { rec }}$ and $\boldsymbol{F}_{C m}^{M, \text { rec }}$ represent the receiving patterns of the $m$ th testing function with respect to a close point $C$ for EFIE and MFIE, respectively. Similarly, $F_{C^{\prime} n}^{E, \text { rad }}$ and $\boldsymbol{F}_{C^{\prime} n}^{M, \text { rad }}$ are the radiation patterns of the $n$th basis function with respect to a close point $C^{\prime}$. The translation function in (23) evaluates the interaction between the basis and testing groups that are located at $C^{\prime}$ and $C$, respectively, and separated by

$$
D=|D| \hat{D}=r_{C}-r_{C^{\prime}} .
$$

As an example, we consider the LL functions of the first kind and take only the first triangles of the basis and testing functions to derive the radiation and receiving patterns as follows:

EFIE and MFIE radiation patterns:

$$
\begin{aligned}
\boldsymbol{F}_{C^{\prime} n 1}^{E, \mathrm{rad}}(\hat{\boldsymbol{k}})= & \int_{S_{n 1}} d \boldsymbol{r}^{\prime} e^{-i \boldsymbol{k} \cdot\left(\boldsymbol{r}^{\prime}-\boldsymbol{r}_{C^{\prime}}\right)}(\boldsymbol{I}-\hat{\boldsymbol{k}} \hat{\boldsymbol{k}}) \cdot \boldsymbol{b}_{n}\left(\boldsymbol{r}^{\prime}\right) \\
= & \frac{l_{n}}{4\left(A_{n 1}\right)^{2}}(\boldsymbol{I}-\hat{\boldsymbol{k}} \hat{\boldsymbol{k}}) \cdot\left(\boldsymbol{r}_{3}-\boldsymbol{r}_{1}\right)\left[\left(\boldsymbol{r}_{4}-\boldsymbol{r}_{1}\right) \times \hat{\boldsymbol{n}}\right] \\
& \cdot \int_{S_{n 1}} d \boldsymbol{r} e^{-i \boldsymbol{k} \cdot\left(\boldsymbol{r}^{\prime}-\boldsymbol{r}_{C^{\prime}}\right)}\left(\boldsymbol{r}^{\prime}-\boldsymbol{r}_{1}\right) \\
= & \boldsymbol{F}_{C^{\prime} n 1}^{M, \mathrm{rad}}(\hat{\boldsymbol{k}})
\end{aligned}
$$

EFIE receiving pattern:

$$
\begin{aligned}
\boldsymbol{F}_{C m 1}^{E, \mathrm{rec}}(\hat{\boldsymbol{k}})= & \int_{S_{m 1}} d \boldsymbol{r} e^{i \boldsymbol{k} \cdot\left(\boldsymbol{r}-\boldsymbol{r}_{C}\right)}(\boldsymbol{I}-\hat{\boldsymbol{k}} \hat{\boldsymbol{k}}) \cdot \boldsymbol{t}_{m}(\boldsymbol{r}) \\
= & \frac{l_{m}}{4\left(A_{m 1}\right)^{2}}(\boldsymbol{I}-\hat{\boldsymbol{k}} \hat{\boldsymbol{k}}) \cdot\left(\boldsymbol{r}_{3}-\boldsymbol{r}_{1}\right)\left[\left(\boldsymbol{r}_{4}-\boldsymbol{r}_{1}\right) \times \hat{\boldsymbol{n}}\right] \\
& \cdot \int_{S_{m 1}} d \boldsymbol{r} e^{i \boldsymbol{k} \cdot\left(\boldsymbol{r}-\boldsymbol{r}_{C}\right)}\left(\boldsymbol{r}-\boldsymbol{r}_{1}\right) \\
= & {\left[\boldsymbol{F}_{C m 1}^{E, \mathrm{rad}}(\hat{\boldsymbol{k}})\right]^{*} }
\end{aligned}
$$

MFIE receiving pattern:

$$
\begin{aligned}
\boldsymbol{F}_{C m}^{M, \text { rec }}(\hat{\boldsymbol{k}})= & -\hat{\boldsymbol{k}} \times \int_{S_{m}} d \boldsymbol{r} e^{i \boldsymbol{k} \cdot\left(\boldsymbol{r}-\boldsymbol{r}_{C}\right)} \boldsymbol{t}_{m}(\boldsymbol{r}) \times \hat{\boldsymbol{n}} \\
= & -\frac{l_{m}}{4\left(A_{m 1}\right)^{2}} \hat{\boldsymbol{k}} \times\left[\left(\boldsymbol{r}_{3}-\boldsymbol{r}_{1}\right) \times \hat{\boldsymbol{n}}\right] \\
& \cdot\left[\left(\boldsymbol{r}_{4}-\boldsymbol{r}_{1}\right) \times \hat{\boldsymbol{n}}\right] \cdot \int_{S_{m 1}} d \boldsymbol{r} e^{i \boldsymbol{k} \cdot\left(\boldsymbol{r}-\boldsymbol{r}_{C}\right)}\left(\boldsymbol{r}-\boldsymbol{r}_{1}\right) .
\end{aligned}
$$

In (26), the superscript “ $*$ " denotes complex conjugation and the final equality holds when the sets of basis and testing functions are the same according to a Galerkin scheme. In (25)-(27), the integrals are evaluated analytically. These integrals are the same as those involved in the RWG implementations. The derivations related to the second triangles and for the LL functions of the second kind are similar.

\section{REFERENCES}

[1] R. F. Harrington, Field Computation by Moment Methods. New York: Macmillan, 1968.

[2] S. M. Rao, D. R. Wilton, and A. W. Glisson, "Electromagnetic scattering by surfaces of arbitrary shape," IEEE Trans. Antennas Propag., vol. AP-30, pp. 409-418, May 1982.

[3] A. J. Poggio and E. K. Miller, "Integral equation solutions of three-dimensional scattering problems," in Comput. Techniques for Electromagnetics, R. Mittra, Ed. Oxford: Permagon Press, 1973, ch. 4.

[4] Ö Ergül and L. Gürel, "Investigation of the inaccuracy of the MFIE discretized with the RWG basis functions," in Proc. IEEE Antennas and Propagation Soc. Int. Symp., 2004, vol. 3, pp. 3393-3396.

[5] L. Gürel and Ö. Ergül, "Singularity of the magnetic-field integral equation and its extraction," IEEE Antennas Wireless Propag. Lett., vol. 4, pp. 229-232, 2005.

[6] Ö. Ergül and L. Gürel, "Improved testing of the magnetic-field integral equation," IEEE Microw. Wireless Comp. Lett., vol. 15, no. 10, pp. 615-617, Oct. 2005.

[7] Ö. Ergül and L. Gürel, "Solid-angle factor in the magnetic-field integral equation," Microw. Opt. Technol. Lett., vol. 45, no. 5, pp. 452-456, Jun. 2005.

[8] Ö. Ergül and L. Gürel, "Improving the accuracy of the MFIE with the choice of basis functions," in Proc. IEEE Antennas and Propagation Soc. Int. Symp., 2004, vol. 3, pp. 3389-3392.

[9] Ö. Ergül and L. Gürel, "The use of curl-conforming basis functions for the magnetic-field integral equation," IEEE Trans. Antennas Propag. vol. 54, pp. 1917-1926, Jul. 2006.

[10] E. Úbeda and J. M. Rius, "Curl-conforming MFIE in the analysis of perfectly conducting sharply-edged objects," in Proc. IEEE Antennas and Propagation Soc. Int. Symp., 2004, vol. 4, pp. 4052-4055.

[11] E. Úbeda and J. M. Rius, "MFIE MoM-formulation with curl-conforming basis functions and accurate kernel integration in the analysis of perfectly conducting sharp-edged objects," Microw. Opt. Technol. Lett., vol. 44, no. 4, pp. 354-358, Feb. 2005.

[12] E. Úbeda and J. M. Rius, "Monopolar divergence-conforming and curl-conforming low-order basis functions for the electromagnetic scattering analysis," Microw. Opt. Technol. Lett., vol. 46, no. 3, pp. 237-241, Aug. 2005.

[13] E. Úbeda and J. M. Rius, "Novel monopolar MFIE MoM-discretization for the scattering analysis of small objects," IEEE Trans. Antennas Propag., vol. 54, pp. 50-57, Jan. 2006.

[14] L. C. Trintinalia and H. Ling, "First order triangular patch basis functions for electromagnetic scattering analysis," J. Electromagn. Waves Applicat., vol. 15, no. 11, pp. 1521-1537, 2001.

[15] Ö. Ergül and L. Gürel, "Improving the accuracy of the magnetic field integral equation with the linear-linear basis functions," Radio Sci., vol. 41, 2006.

[16] J. Song, C.-C. Lu, and W. C. Chew, "Multilevel fast multipole algorithm for electromagnetic scattering by large complex objects," IEEE Trans. Antennas Propag., vol. 45, pp. 1488-1493, Oct. 1997.

[17] A. F. Peterson, S. L. Ray, and R. Mittra, Computational Methods for Electromagnetics. New York: IEEE Press, 1998. 
[18] R. D. Graglia, D. R. Wilton, and A. F. Peterson, "Higher order interpolatory vector bases for computational electromagnetics," IEEE Trans. Antennas Propag., vol. 45, pp. 329-342, Mar. 1997.

[19] J. Wang and J. P. Webb, "Hierarchal vector boundary elements and p-adaptation for 3-D electromagnetic scattering," IEEE Trans. Antennas Propag., vol. 45, pp. 1869-1879, Dec. 1997.

[20] W. Cai, T. Yu, H. Wang, and Y. Yu, "High-order mixed RWG basis functions for electromagnetic applications," IEEE Trans. Microw. Theory Tech., vol. 49, pp. 1295-1303, Jul. 2001.

[21] L. Gürel and Ö. Ergül, "Comparisons of FMM implementations employing different formulations and iterative solvers," in Proc. IEEE Antennas and Propagation Soc. Int. Symp., 2003, vol. 1, pp. 19-22.

[22] J. R. Mautz and R. F. Harrington, "H-field, E-field, and combined field solutions for conducting bodies of revolution," $A E \ddot{U}$, vol. 32, no. 4, pp. 157-164, Apr. 1978.

[23] C. P. Davis and K. F. Warnick, "High-order convergence with a low-order discretization of the 2-D MFIE," IEEE Antennas Wireless Propag. Lett., vol. 3, pp. 355-358, 2004.

[24] C. P. Davis and K. F. Warnick, "2D MFIE solution improvement by regularization," in Proc. IEEE/ACES Int. Conf., 2005, pp. 321-324.

[25] W. C. Chew, J.-M. Jin, E. Michielssen, and J. Song, Fast and Efficient Algorithms in Computational Electromagnetics. Boston, MA: Artech House, 2001.

[26] R. D. Graglia, "On the numerical integration of the linear shape functions times the 3-D Green's function or its gradient on a plane triangle," IEEE Trans. Antennas Propag., vol. 41, pp. 1448-1455, Oct. 1993.

[27] R. E. Hodges and Y. Rahmat-Samii, "The evaluation of MFIE integrals with the use of vector triangle basis functions," Microw. Opt. Technol. Lett., vol. 14, no. 1, pp. 9-14, Jan. 1997.

[28] P. Y.-Oijala and M. Taskinen, "Calculation of CFIE impedance matrix elements with RWG and $\hat{\boldsymbol{n}} \times$ RWG functions," IEEE Trans. Antennas Propag., vol. 51, pp. 1837-1846, Aug. 2003.

[29] D. A. Dunavant, "High degree efficient symmetrical Gaussian quadrature rules for the triangle," Int. J. Numer. Meth. Eng., vol. 21, pp. $1129-1148,1985$.

[30] Ö. Ergül, "Fast multipole method for the solution of electromagnetic scattering problems," M.S., Bilkent Univ., Ankara, Turkey, 2003.

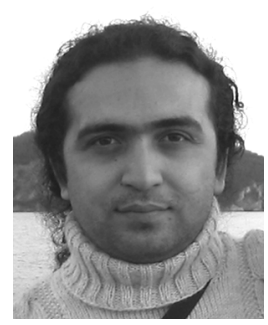

Özgür Ergül (S'98) was born in Yozgat, Turkey, in 1978. He received the B.S. and M.S. degrees in electrical and electronics engineering from Bilkent University, Ankara, Turkey, in 2001 and 2003, respectively. He is currently pursuing the Ph.D. degree at Bilkent University.

Since 2001, he has served as a Teaching and Research Assistant in the Department of Electrical and Electronics Engineering at Bilkent University. From 2000 to 2005 , he was affiliated with the Computational Electromagnetics Group at Bilkent University and, since 2005, with the Computational Electromagnetics Research Center (BiLCEM). His research interests include fast and accurate algorithms for the solutions of large and complicated structures, integral equations, parallel programming, and iterative techniques.

Mr. Ergül's academic endeavors are supported by the Scientific and Technical Council of Turkey (TUBITAK) in the framework of a national Ph.D. scholarship.

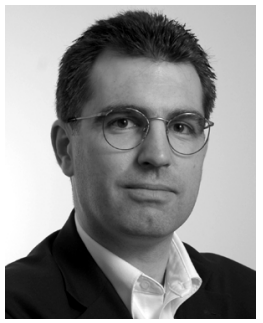

Levent Gürel (S'87-M'92-SM'97) received the B.Sc. degree from the Middle East Technical University (METU), Ankara, Turkey, in 1986, and the M.S. and $\mathrm{Ph} . \mathrm{D}$. degrees from the University of Illinois at Urbana-Champaign (UIUC) in 1988 and 1991, respectively, all in electrical engineering.

He joined the Thomas J. Watson Research Center of the International Business Machines Corporation, Yorktown Heights, New York, in 1991, where he worked as a Research Staff Member on the electromagnetic compatibility (EMC) problems related to electronic packaging, on the use of microwave processes in the manufacturing and testing of electronic circuits, and on the development of fast solvers for interconnect modeling. Since 1994, he has been a faculty member in the Department of Electrical and Electronics Engineering of the Bilkent University, Ankara, where he is currently a Professor. He was a Visiting Associate Professor at the Center for Computational Electromagnetics (CCEM) of the UIUC for one semester in 1997. He returned to the UIUC as a Visiting Professor in 2003-2005, and as an Adjunct Professor in 2005-2006. He founded the Computational Electromagnetics Research Center (BiLCEM) at Bilkent University in 2005, where he is serving as the Director. His research interests include the development of fast algorithms for computational electromagnetics (CEM) and the application thereof to scattering and radiation problems involving large and complicated scatterers, antennas and radars, frequency-selective surfaces, high-speed electronic circuits, optical and imaging systems, nanostructures, and metamaterials. He is also interested in the theoretical and computational aspects of electromagnetic compatibility and interference analyses. Ground penetrating radars and other subsurface scattering applications are also among his research interests.

Prof. Gürel has received much recognition for his accomplishments, including an award from the Turkish Academy of Sciences (TUBA) in 2002 and the Scientific and Technical Research Council of Turkey (TUBITAK) in 2003. He has served as the Chairman of the AP/MTT/ED/EMC Chapter of the IEEE Turkey Section in 2000-2003. He founded the EMC Chapter in Turkey in 2000. He served as the cochairman of the 2003 IEEE International Symposium on Electromagnetic Compatibility. He is a member of the General Assembly of the European Microwave Association, a member of the USNC of the International Union of Radio Science (URSI), and the chairman of Commission E (Electromagnetic Noise and Interference) of URSI Turkey National Committee. He is currently serving as an associate editor of Radio Science. 